\title{
Mhcj

\section{El uso del cortometraje como estrategia de branded content}

\author{
Dra. María Dolores Lorán Herrero| mdloran@ucam.edu \\ Universidad Católica de Murcia
}

\author{
Palabras clave \\ Cortometraje; Comunicación Audiovisual; \\ Comunicación Corporativa; Branded Content; \\ Comunicación Organizacional e Innovación. \\ Sumario \\ 1. Introducción 2. El branded content \\ 2.1. La ficción en el branded content \\ 2.2. El cortometraje en el branded content 3. \\ Metodología 4. Clasificación \\ 5. Discusión y conclusiones 6 . Referencias
}

\section{Resumen}

La inclusión del cortometraje como fórmula de branded content está siendo utilizada por las empresas como estrategia asociada a la búsqueda de la generación de contenido de marca. La experiencia de las empresas españolas, en este sentido, es todavía escasa, pero existen cada vez más organizaciones que centran el posicionamiento de sus marcas a través de fórmulas de este tipo. El cortometraje aparece con fuerza en la búsqueda de nuevas maneras de posicionar en la mente de los públicos, de una manera sutil, una conexión emocional y un valor añadido.

Los nuevos cortometrajes como los realizados por Alberto Rodríguez "Las pequeñas cosas" para Estrella Damm y por Javier Fesser "17 años juntos" para ING Direct, corroboran esta nueva tendencia $y$, aunque la utilización empresarial que se da a esta fórmula audiovisual no es la habitual, se produce una perfecta simbiosis entre los dos ámbitos, lo que hace necesario su estudio en profundidad.

El objetivo de esta comunicación es conocer el uso actual que se da al cortometraje y su proyección de cara a las empresas implicadas en la utilización de esta fórmula. Para ello se ha realizado una revisión bibliográfica aplicada al documento audiovisual y centrada en el cortometraje. La conclusión a la que se ha llegado es, el aumento de las experiencias audiovisuales en el mundo de la empresa destacando el posicionamiento del cortometraje como una de los principales técnicas de branded content.

\section{Cómo citar este texto:}

María Dolores Lorán Herrero (2017): “ El uso del cortometraje como estrategia de branded content”, en Miguel Hernández Communication Journal, nº, pp. 153 a 177. Universidad Miguel Hernández, UMH (Elche-Alicante). Recuperado el __ de de: [link del artículo en mhjournal.org] de 20 


\title{
The use of the short film as a branded content strategy
}

\author{
Dra. María Dolores Lorán Herrero | mdloran@ucam.edu \\ Universidad Católica de Murcia
}

\author{
Keywords \\ Film Short, Audiovisual Communication, \\ Corporative communication, Branded Content, \\ Organizational Communication and Innovation \\ Summary \\ 1. Introduction 2. Branded content \\ 2.1. Fiction in the branded content \\ 2.2. The short film in the branded content 3. \\ Methodology 4. Classification \\ 5. Discussion and conclusions 6. References
}

\begin{abstract}
The inclusion of short films as a branded content formula is being used by companies as a strategy associated with the search for the creation and promotion of branded content. In this sense, the experience of Spanish companies is still scarce, but organizations that focus on positioning their brands through these kinds of new formulas have increased. Short films show up firmly in search of new methods of establishing a subtle emotional link and a value added in the consumer mindset
\end{abstract}

The new short films like "Little things" for Estrella Damm, by Alberto Rodríguez, or "17 years together" for ING Direct, by Javier Fesser, support this new trend although business use of audiovisual formula is not so common. However, there is an ideal symbiosis between these two areas and a research about this phenomenon turns out to be necessary for the current academics debates.

The purpose of this communication is to know how business and companies use short films as a communication formula nowadays. To get this aim, we have applied a literature review methodology about audiovisual documents and short films. The main conclusion is that audiovisual experiences in the business sphere have increased and short films are one of the most important branded content tools.

\section{How to cite this text:}

María Dolores Lorán Herrero (2017): “The use of the short film as a branded content strategy", en Miguel Hernández Communication Journal, nº, pp. 153 to 177. Universidad Miguel Hernández, UMH (Elche-Alicante). Accessed 20_ in: [paper link in mhjournal.org] 


\section{Introducción}

El branded content está considerado como una de las estrategias de marketing más efectiva en la búsqueda de la creación de contenido de marcas. Desde el punto de vista de la comunicación corporativa audiovisual y, entendiendo que se asocia a una técnica, algunos autores (Regueira, 2011; Renedo y Alonso del Barrio, 2014; De Aguilera-Moyano, Baños-González y Ramírez-Perdiguero, 2015) consideran el branded content como una fórmula en sí misma con unas características determinadas que posicionan la marca en la mente de los públicos. Una de las referencias principales que se le otorga en este sentido, es el uso de la imagen y el sonido como elementos principales para lograr su objetivo publicitario. Carmen Costa-Sánchez (2015) lo denomina Audiovisual Branded Content al asociarlo a plataformas en las que se utiliza contenido audiovisual, aunque sea con multitud de fórmulas variadas.

La publicidad convencional muestra una pérdida de eficacia y se ha producido un cambio sustancial en el medio tradicional con mayor inversión publicitaria: la televisión, donde el modelo de negocio está cambiando. La pérdida de eficacia se produce por el cansancio de los públicos por los mensajes intrusivos que no desean ver y por los cambios en el ecosistema de medios donde el avance de la tecnología provoca la convergencia entre el entretenimiento y otros sectores según Gambetti y Grafigna (2010) tal y como destacan De Aguilera-Moyano, Baños-González y Ramírez-Perdiguero (2015: 522). Ya no se interrumpe a los consumidores con anuncios, sino que se les atrae con mensajes útiles, entretenidos o relevantes, para que estos decidan acceder libremente a los contenidos e incluso compartirlos con otras personas, sobre todo, cuando se recurre a la emoción o la sorpresa. Las marcas destinan parte de sus recursos al marketing experiencial con el que, además de comunicar, conectan la oferta con experiencias únicas en la búsqueda de ese engagement que forme una comunidad perfectamente identificada e involucrada con la marca.

$\mathrm{El}$ auge de los formatos below the line, es decir, los no convencionales, como los denomina Mónica Matellanes Lazo (2014: 319), son cada vez más atractivos para los anunciantes, ya que así diferencian más sus marcas. Estas fórmulas impactan más en el usuario y permiten memorizar mejor el mensaje y, sobre todo, incidir en el recuerdo de la marca. 


\section{El branded content}

La base del branded content se encuentra en "ofrecer experiencias, no anuncios" (Regueira, 2011) tal y como destacan Cristina del Pino-Romero y Araceli Castelló-Martínez (2015). Se trata de una oportunidad: la de generar contenido de marca sin importar el tipo de formato. Tiene como objetivo la creación de un contenido útil y que genere un valor añadido a la marca. Si captar la atención del usuario a través del contenido es complejo, ya que hay mucho, la dificultad de destacar se incrementa. Productos como el fashion film, el branded film y los cortometrajes entre otros, aparecen como fórmulas que se distinguen en su nomenclatura, pero que coinciden en su nexo, en su fondo: mejorar la imagen de marca, tal y como indican Renedo y Alonso del Barrio (2014). Para estos autores, "ofrecer contenidos interesantes y útiles a tus clientes y potenciales clientes para acercarte a ellos en lugar de gritarles que compren tus productos o servicios, se convierte en una táctica más efectiva que la publicidad directa" (2014:7)

Uno de los elementos más novedosos que aporta esta fórmula es que las marcas escuchan a los consumidores y estos son quienes ostentan el poder, tal y como lo refleja la revista digital The Social Media Family. Sin perder de vista el sentido comercial que toda estrategia de marketing debe tener en cuenta, la compra final de un producto, es cierto que el objetivo principal, la venta, ahora se comparte con la fidelización del cliente, generalmente buscando la simpatía hacia la marca. Se fomentan acciones de comunicación, en la mayoría de las ocasiones, que pretenden ser divertidas y entretenidas. Se intenta que el propio consumidor busque la publicidad y no al contrario. Según los datos que aportan en el documento, y que han sido extraídos de Content Scope 2015 (estudio de medición, tendencias y uso del Branded Content en España), esto no es una moda pasajera y siete de cada diez anunciantes ya lo hacen, lo que supone un aumento de veintidós puntos porcentuales respecto al año 2013.

Tradicionalmente se han utilizado dos grandes tipos de acciones de comunicación: pull y push tal y como explican J de Aguilera-Moyano, BañosGonzález y Ramírez-Perdiguero. Estas categorías se utilizan para diferenciar las herramientas de comunicación por su nivel de agresividad o de relación: "las push fuerzan al consumidor a exponerse a los mensajes, son agresivas e intrusivas, como sucede con la publicidad convencional; en las acciones pull el consumidor decide si accede a los contenidos de marca" (2015: 521). Inciden por ello en que en el nuevo contexto, el marketing push está en declive y las acciones pull parecen las más adecuadas para llegar a un consumidor que gestione el acceso a los contenidos que desea y así controlar su relación con las 
marcas. Los consumidores son cada vez más reticentes a la publicidad convencional orientada a una venta directa y se decantan por un modelo que les permita acceder a ella en el momento que desee.

Según el documento elaborado por Corporate Excellence, Centre por Reputación Leadership "tendencias en la generación de contenido de marca: Branded Content", no se trata sólo de un tipo de técnica de comunicación de marca, sino que los soportes son varios, incluyendo el audiovisual que es el mayoritario:

1. Audiovisual, series de televisión, reality shows, programas cortos, patrocinados, TV en directo y canales on-line de televisión.

2. Digital: plataformas sociales, experiencias interactivas, dispositivos móviles y webs de experiencia.

3. Eventos: deportivos, cine o simulaciones de guerrilla.

4. Juegos: advergames, aplicaciones móviles, etc.

5. Música: artistas esponsorizados, conciertos en directo o videoclips musicales.

6. Impresos: revistas y libros.

Los diversos soportes sirven para hacer partícipes a los consumidores, ya sea vía $V \log$, redes o aplicaciones, donde el fin es que llegue a sus manos, haciendo del participante el centro de la acción comunicativa. Técnicas como el branded content no sólo permiten una comunicación efectiva de marca, sino también suponen un apoyo económico a proyectos audiovisuales, ya que como afirman del Pino Romero y Castelló Martínez (2015), los contenidos, especialmente los audiovisuales, se han convertido en la piedra angular para generar interés y atención. Se transforman en espacios de información o entretenimiento con contenidos audiovisuales creados por y para una marca. Costa-Sánchez (2015) distingue tres tipos: el de entretenimiento, el informativo y el educativo. Según esta autora, la práctica está infrautilizada en las empresas españolas, pero se constata un aumento significativo en los últimos tiempos. La notoriedad y la interacción con el consumidor destacan como valores añadidos a una propuesta de branded content, si se comparan con otras acciones de comunicación más usuales. Se ha pasado de la comunicación bidireccional a una comunicación en red que facilitan la creación y distribución de contendidos. La aparición de la marca es menos agresiva y más duradera que en la publicidad tradicional y aprovechando un eje narrativo específico, se comunican valores diferenciales de tal manera que resulten atractivos y no intrusivos al ciudadano. Esta fórmula adquiere todavía mayor valor cuando comienzan a entender el potencial del storytelling (Jenkins, 2008; Scolari, 2013 y Grandío, 2015), es decir, el uso del relato para mostrar el espíritu de una marca. 
Siguiendo esta línea aparece un tipo de mensaje híbrido que J de AguileraMoyano, Baños-González y Ramírez-Perdiguero (2015) relacionan con el que las marcas producen como contenidos de entretenimiento: el branded entertainment con un amplio abanico de posibilidades y con una perspectiva de crecimiento muy amplia y adecuada para generar engagement. Se trata de una estrategia que cambia de sitio al producto y al consumidor, dejando a este en el centro ya que se convierte en la fuerza motriz que está detrás de su comportamiento y de la toma de decisiones (De Aguilera-Moyano, BañosGonzález y Ramírez-Perdiguero, 2015). A los nuevos modelos de marketing se les unen hábitos de consumo de medios muy distintos a los de hace unos años. Los mensajes híbridos, como indican estos autores, combinan el mensaje comercial de una marca con un contenido no comercial capaz de proporcionar una experiencia positiva. Incluyen acciones comunicativas pagadas por el anunciante con el objetivo de influir en el público y así obtener un beneficio comercial, recurriendo a fórmulas de comunicación de carácter no exclusivamente comercial.

La ventaja de estas fórmulas es que "el espectador las recibe con mayor atención y receptividad, no atribuyendo su presencia a una acción de comunicación del anunciante sino a las características del contenido de entretenimiento que ha decidido consumir" (De Aguilera-Moyano, BañosGonzález y Ramírez-Perdiguero, 2015: 523). Se consigue un acceso voluntario a un contenido satisfactorio, que produce un nexo con el anunciante que no se crea con la publicidad tradicional. La marca se integra en el contenido de una manera natural y transmite una serie de valores que van calando en el consumidor a través de la emoción, la diversión, el entretenimiento o la sorpresa.

\subsection{La ficción en el branded content.}

Memorizar el mensaje y buscar una relación con la marca son cuestiones en las que se apoya la estrategia del uso del cortometraje ya que se produce una perfecta simbiosis entre las dos fórmulas. Como precisan en la revista digital Antevenio.com, mediante historias impactantes y contenidos que no tienen por qué ver con la actividad mercantil de la empresa, las marcas pretenden generar notoriedad y afinidad con sus clientes. El objetivo es dar a conocer sus valores, además de emocionar y apelar a los sentimientos de los clientes. Se trata de generar compromiso respecto a la marca porque se acercan así al usuario (Costa-Sánchez, 2015). La marca es una parte más de lo que se promociona o anuncia. Se va más allá de la experiencia de la publicidad. Se anuncia todo lo que rodea a la marca, lo que la construye, lo que hace que la 
marca sea como es. El emplazamiento y el ambiente se hacen protagonistas de la sensación que se transmite al espectador. En este sentido, el cortometraje se postula como uno de los mejores medios para conseguirlo.

En el medio cinematográfico cabe destacar como uno de los branded content más interesantes, la película "Solo se vive una vez" (Zindagi Na Milegi Dobara, 2011), producción de Bollywood rodada en España que ha tenido una gran repercusión entre la sociedad india y que ha ayudado a impulsar el turismo nacional. La película, que se llevó siete premios en Filmfare (los Oscar de Bollywood), narra un viaje de tres amigos por España: los Sanfermines de Pamplona, Sevilla, Barcelona y la Tomatina de Buñol son algunos de los lugares que visitan. Fue vista en el cine por más de 70 millones de personas en India y la cifra de indios que han visitado este país ha pasado de unos 70.000 a casi 200.000, con un perfil joven, siguiendo la estela de los protagonistas. El gobierno español, por medio de su ente público TurEspaña, llegó a un acuerdo con la productora del filme Excel Entertainment, por el cual TurEspaña prestó una ayuda económica a cambio de promocionar la imagen del país. De hecho, antes del filme, se incluye el spot publicitario "You need Spain". En 2014, otro destacado ejemplo de branded content llegó a las pantallas del cine: "The Lego Movie". Su estreno en Estados Unidos alcanzó una recaudación de más de 69 millones de dólares, convirtiéndose en el segundo mejor estreno de un mes de febrero. Tras la película, Lego creó un videojuego basado en las aventuras narradas en la misma y ha dado continuidad a esta acción con productos como juguetes inspirados en los personajes y merchandising, tal y como describen Cristina del Pino-Romero y Araceli Castelló-Martínez (2015).

La dificultad en la definición y usos de la ficción como estrategia de branded content radica en ubicar la sutil línea que diferencia fórmulas como el branded entertainment, el ya aludido product placement, el fashion film e incluso la aparición de la marca en webseries, teaser, promos de televisión, el advertainment e incluso el advergaming. Todas ellas pertenecen a fórmulas de promoción y marketing que pueden tener características comunes como la aparición de la marca, más o menos visible y constatable, pero son diferentes en su forma de realización.

Otra característica común es el uso de la ficción como base para sus productos, sin ser películas en todos los casos, sí que podrían nutrirse de historias ficcionadas muy cercanas al cortometraje, incluso como falsos cortometrajes. La mezcla en el mundo audiovisual se refleja "en la fusión de intereses entre el consumo y la ficción”, tal y como precisan Cristina del Pino y Fernando Olivares (2007: 343). La industria del entretenimiento, sobre todo en el sector de la ficción audiovisual, cinematográfica y televisiva, se postula 
como fórmula atractiva y rentable, como precisan del Pino y Olivares (2007), sobre todo, porque sirve como instrumento a las dos áreas que participan de estas estrategia: la marca y el sector audiovisual que, con su fusión, atienden a los objetivos tanto comerciales como corporativos.

El mix de fórmulas corporativas audiovisuales que se nutren de la ficción aumenta considerablemente y está en constante evolución lo que dificulta aún más su revisión bibliográfica. La ficción audiovisual aparece para ayudar a que las marcas busquen su protagonismo y comuniquen los valores de la empresa de muy diversas formas. La palabra "integración" sirve para definir esa mezcla de disciplinas como son la comunicación comercial, la corporativa y la comunicación audiovisual. En este contexto se defiende el término Comunicación Corporativa Audiovisual (Galindo, 2004, 2005; Costa-Sánchez, 2014; Lorán-Herrero, 2012, 2016) que las empresas poco a poco introducen en su ideario como estrategia para potenciar su imagen de marca a través de diversas fórmulas audiovisuales. Los films y los cortometrajes se convierten en contenedores donde pueden aparecer las marcas y, por ello, estas se decantan por la ficción. La influencia de la industria audiovisual y del entretenimiento en la sociedad actual, potenciada por la facilidad de acceso que supone Internet, permite que las empresas apuesten por el cine y sus productos, y el cortometraje se erige como una de las fórmulas que sin llegar a necesitar el alto presupuesto de una película, puede transmitir en un espacio de tiempo bastante más breve, la esencia que una marca desea trasladar a sus stakeholders. Se produce y, de hecho, se potencia lo que Cristina del Pino y Fernando Olivares ya definían como "la comunicación global al servicio del marketing fílmico” (2007: 347). En la extensa revisión fílmica que proponen en su artículo, detectan que más que una intencionalidad comercial lo que existe es un propósito corporativo.

De la intención corporativa arriba mencionada por Del Pino y Olivares (2007), es de donde se nutren las actuales estrategias que las empresas e instituciones desarrollan actualmente: promocionales de televisión, teaser, webserie, advertainment, advergaming $\mathrm{y}$, por supuesto, películas y cortometrajes. La exposición evidente de la marca desaparece en pro de ser un marco de actuación o un elemento que apoye una acción determinada en cualquier fórmula de ficción. Se amplían los horizontes con el binomio marca-ficción y así, los creadores de ficción ven a a las empresas y a sus marcas como una vía de ingresos para optimizar sus beneficios. Los proyectos audiovisuales nacen de la mano de un partner con una presencia plana en la mayoría de las ocasiones, sólo una sutil referencia y, como precisan Del Pino y Olivares "el poder de la influencia de la ficción audiovisual atrae a las marcas” (2007: 351), 
lo que permite ofrecer un panorama abierto de posibilidades que sólo la creatividad puede ir marcando.

\subsection{El cortometraje en el branded content}

Los cortometrajes forman parte, cada vez más, de los ejemplos de Branded Content porque su alto nivel creativo destaca como estrategia de valor hacia la marca. A diferencia de otros productos similares, que se apoyan en estrategias relacionadas con el product placement tradicional, la marca apenas se manifiesta visualmente y, si lo hace, es de una manera sutil (Rodríguez, Gil y Marzal, 2015: 143). La conexión entre la marca con el mundo del cine se ha mostrado en multitud de ocasiones, tal y como precisan estos autores. Al inicio, la marca destinó sus estrategias hacia los campos de la exhibición y la distribución, buscando una conexión más espacial que sensitiva. Así es como se aborda actualmente, con la búsqueda de experiencias generalmente positivas, aunque en el estudio que estos autores realizan sobre la marca Gas Natural Fenosa, se encuentran con un dilema comunicativo, ya que por un lado, la empresa pretende proporcionar a su audiencia un interés hacia su responsabilidad corporativa (centrada en el medio ambiente, la eficiencia energética y el consumo responsable) y, por otro lado, los cortometrajes de la campaña de branded content analizada, la realizan tres directores de cine de terror, lo que garantiza mensajes distópicos, sádicos y, a priori, radicalmente opuestos a la trayectoria publicitaria de la propia marca. Encuentran la razón probablemente en una metáfora en torno a la crisis económica con base en una relación resbaladiza y sin conexión con la actividad real de la marca.

El cortometraje destaca como el producto más efectivo para llevar a cabo las diversas estrategias que se están desarrollando en la actualidad. Según De

Aguilera-Moyano, Baños-González y Ramírez-Perdiguero (2015), el cortometraje es el formato más atractivo y la plataforma de visionado más utilizada es Internet; eso sí, sin olvidar que los consumidores deben ser conscientes de la existencia de un contenido determinado y así tomar la decisión de consumirlo o no. Por este motivo, proliferan estrategias basadas en narraciones audiovisuales ficcionales que buscan presentar a la marca y conectar con la audiencia. El consumidor, antes influenciable, se convierte en crítico activo para la marca. Se trata de acudir al prosumidor que, en el caso del acceso al cortometraje, resulta imprescindible. Los contenidos deben resultar atractivos y reforzar los valores y el mensaje corporativo diseñado por la marca. 
Una de las características que identifican al cortometraje, utilizado como branded content, es que su consumo puede realizarse de manera autónoma, es decir, independientemente de la marca, se puede disfrutar como producto único, con su historia y narración por sí mismo y, por eso, se definen por lo que Caerols Mateo y De la Horra Veldman denominan "hibridación" (2015: 341). El consumo de productos que obedecen a códigos ya conocidos, pero que se utilizan con fines distintos, proporciona a estos un aura de fórmula que mezcla lo convencional con lo novedoso y que puede utilizarse en ambos contextos sin que en ningún momento parezca extraño su uso. El consumidor habitual de cortometrajes, al igual que un seguidor de las redes sociales, se convierten en usuarios potenciales y figuras imprescindibles. Se preguntan estos autores cuál es el papel que ocupan los profesionales de la comunicación en este tipo de fenómenos y, es en este momento, en el que se debe "poner de relieve que las audiencias (que podemos traducir en seguidores) se individualizan como nunca lo habían hecho" (Caerols Mateo y De la Horra Veldman, 2015: 347). Esto resulta determinante para entender que a cada individuo se le presenta la opción de generar y acceder a los contenidos que desee.

Esta opción apunta en una nueva dirección que los profesionales de la comunicación deben plantearse y es que, además de que los contenidos se pueden encontrar en diversos lugares o plataformas, las ofertas deben personalizarse. Los cortometrajes no están exentos a esta situación y por ello deben encontrar la mejor manera para posicionarse. Si Internet permite al usuario común crear contenidos de diversas y cuestionables calidades, lo importante ahora es que la exigencia de la profesionalización de estrategias que utilicen el cortometraje para las marcas, sea del mayor nivel posible para conseguir distinguirse. Los intangibles de la marca, que se muestran en estas fórmulas audiovisuales, deben estar gestionadas por profesionales de todos los ámbitos de la comunicación en la búsqueda de su eficiencia. El hecho de que Internet y las redes sociales puedan aumentar de forma muy considerable la cantidad de productos que se pueden visionar, dificulta la tarea de los profesionales de la comunicación. Como ya indicaba Hipólito Vivar (2011), se origina un proceso de integración y renovación que aunque no está exento de dificultad, abre un amplio abanico de posibilidades. Según Vivar, "las empresas de servicios de marketing y publicidad se enfrentan a un mercado muy dinámico que tiende cada vez más a la gestión integral de sus marcas en los nuevos canales y soportes digitales, sin olvidar los medios tradicionales, que tienen como resultado un gran volumen y una mayor complejidad de sus productos de comunicación” (2011: 3). 
Las estrategias se han ido adaptando al mercado y sus necesidades y, por ello, la gestión de la comunicación pasa por la inclusión de fórmulas, como los cortometrajes, que dinamizan y multiplican el conocimiento de la marca. En el estudio que Eva Reinares-Lara et al (2016) realizan sobre los formatos de publicidad no convencional y su relación con el recuerdo de la marca, destacan que "a igualdad de audiencia y duración, las nuevas formas de publicidad en televisión generan mayor recuerdo que la publicidad convencional (spots)" (2016: 268). Es cierto que el exhaustivo estudio se centra en la televisión, pero hay que destacar una cuestión que estos autores apuntan en sus conclusiones y que parece determinante en el caso de los cortometrajes y su relación con las marcas. Según argumentan, lo óptimo en un plan de comunicación es la combinación de ambos tipos de publicidad. De hecho, varios de los cortometrajes aquí analizados, utilizan esta fórmula combinada y se realiza un spot para televisión que invita al espectador a ver el cortometraje completo en Internet, se trata de "Vale" (2015) y "Las pequeñas cosas" (2016), campañas de branded content realizadas para Estrella Damm.

En la página web de la productora Catorce Producciones se estudia uno de los ejemplos de branded content más exitoso, el de la miniserie producida por Intel y Toshiba y Gran Prix en Cannes 2013, "The beauty inside". Consta de una serie de 6 cortometrajes de 6 minutos cada uno que forman una única historia. A partir de un claim con tantas posibilidades dramáticas, como "The beauty inside", Intel y Toshiba han creado la historia de una persona que se despierta cada día siendo alguien diferente, un día lo hace siendo un hombre de cuarenta años; al siguiente siendo una mujer de sesenta; al siguiente siendo un anciano y así sucesivamente. Lo que se busca es una nueva forma de ficción donde se aprovecha la capacidad de interacción, participación e interactividad que permiten las redes sociales. La campaña se crea con dos marcas, Intel y Toshiba, que se compenetran para mostrarse a través de la ventana Internet. La valentía de esta estrategia radica, a nivel audiovisual, en la importancia de hacer tangible el valor de un microprocesador incluido en un Ultrabook Thosiba, que ayuda a guardar los datos y las experiencias diarias del protagonista. La clave del Branded Content es que una marca crea un universo y una trama y consigue comunicarse con el espectador que, a su vez, interactúa antes de los propios cortometrajes generando contenido para el producto final y convirtiéndose en protagonistas.

Muy relacionados con el cortometraje, ya que ha sido utilizado como base en diversas ocasiones, están los Fashion Film que, como definen Caerols Mateo y De la Horra Veldman, "no son exactamente anuncios, ni cortometrajes, ni vídeos artísticos o musicales, sino una mezcla de ellos, que genera, inevitablemente, nuevos lenguajes narrativos y de experimentación" (2015: 
351). Esta fórmula creativa, que bebe de otras muchas, se ha nutrido de cortometrajes, ya que han comprendido "los beneficios que el movimiento de la imagen puede aportar al producto cultural de una marca. Mostrar algo pero acompañado de historias, sonidos y sentimientos potencia en el cliente un imaginario más fuerte" (2015: 356). Son piezas audiovisuales que beben del vídeo musical, del spot publicitario y del corto de ficción, lo que les hace, en muchas de las ocasiones, parecer claramente un cortometraje. Es el caso de "Lady Blue Shangai"', el cortometraje que David Lynch dirigió en 2010 para la marca de lujo Dior. Protagonizado por Marion Cotillard, el corto nos transporta a una habitación de hotel en la que aparece un misterioso bolso; por supuesto, de Dior. La atmósfera que impregna la pieza -desde la música hasta el guión- es huella inequívoca del estilo sugestivo del director. Otro cortometraje considerado fashion film es el que Roman Polansky produce para Prada, "A Therapy", con contenido propios e interpretado por Helena Bonham Carter y Ben Dingsley. En ambos casos son piezas que representan a marcas de moda y que se exhiben bajo la modalidad de cortometraje.

El incremento del uso que se está haciendo de la ficción como elemento de branded content, por parte de las marcas, es innegable. Con respecto a los contenidos, y más cuando se trata de fórmulas narrativas como el cortometraje, han de estar elaborados y adaptados a los diferentes dispositivos de recepción y pantallas. La marca, dentro del cortometraje, no solo aporta contenido, sino que debe ser parte de la experiencia que se proyecta. En el exhaustivo análisis que realizan Araceli Castelló, Cristina del Pino y Victoria Tur-Viñes (2016) sobre las estrategias que realizan las marcas dirigidas al público adolescente, se denota una clara preferencia por el uso de la ficción en sus más diversas variables (webseries, videoclip, series de televisión, programas de radio y televisión, etc.) y, entre ellos, aparece el cortometraje como una de las modalidades incluidas en su estudio. Es el caso del denominado "La Huida" (2012) protagonizado por Cristina Pedroche y producido por Energy System Soyntec, S.A. que es una empresa de Telecomunicaciones e Internet que opta por esta fórmula; y del cortometraje "La otra película" (2014), creada por Sony Pictures Entertainment, para la marca Sony Pictures España. Se trata de cuatro cortometrajes que recibieron millones de reproducciones en Youtube, ya que sus protagonistas fueron los youtubers Rubius y Mangel. La acción en este caso fue promocional, ya que era parte del estreno en cines de la película "Corazones de acero" (2014). En ambos casos se intenta, no sólo de aportar un contenido de marca, sino de asociarlo a una estrategia transmedia con acciones en web, redes sociales, acceso a información adicional especial, making off, tomas falsas, etc. Para estos autores, el uso de acciones de comunicación, como las recién mencionadas, son un ejemplo "de las peculiaridades de las industrias culturales, en las que el consumidor se erige 
como agente activo y participativo" (Castelló, Del Pino y Tur-Viñes, 2016: 149-150). Los profesionales, indican según conclusiones de su estudio, son conscientes del cambio constante, lo que supone un esfuerzo en diseñar soluciones comunicativas bajo fórmulas narrativas que permitan una distribución multiplataforma.

\section{Metodología}

Una vez realizada una revisión teórica de los términos que influyen conceptualmente en la estrategia del uso del cortometraje y, tras constatar, la perfecta simbiosis que se produce en estos dos ámbitos, lo que se pretende en esta investigación es revisar, mostrar y ordenar el uso de los cortometrajes más determinantes que se han utilizado y se utilizan como estrategia de branded content. Se incluirán además fórmulas de ficción similares y que destacan por su valor estratégico empresarial. Se pretende dividir y organizar la información existente en torno a este tipo de piezas audiovisuales para distinguir y destacar el sentido que se le da y su utilización estratégica. En la investigación realizada se detectan dos maneras de uso bien diferenciadas: de forma autónoma o como parte de una estrategia en la que suele acompañarse de otros cortometrajes. La clasificación tipifica todos los cortometrajes que se han encontrado en la revisión y se han ordenado cronológicamente. Se han incluido tres películas consideradas claros ejemplos de branden content y un falso tráiler. En algunos casos ya se habrá hecho referencia a ellos en el texto por interés sobre el contenido, pero, aún así, se ha decidido volver a incluirlos al considerar que la claridad expositiva es determinante para su tipificación.

\section{Clasificación}

\section{PELÍCULAS:}

- Sólo se vive una vez (Zindagi Na Milegi Dobara, 2011): película producida por Bollywood y rodada en España que ha ayudado a impulsar el turismo nacional en la India, donde tuvo gran repercusión. Promocionada por el ente público TurEspaña, se llegó a un acuerdo económico con la productora Excel Entertainment para promocionar la imagen del país a cambio de una ayuda económica como clara estrategia de promoción de imagen de marca del país.

- VIRAL (2013): dirigida por Lucas Figueroa para FNAC y producida por LMF Films. Fnac ideó una curiosa estrategia basada en una acción de comunicación en la que se buscaba a un "friki para vivir en Fnac". Se buscó a un apasionado de la cultura que se atreviese a vivir diez días en la tienda que la marca tiene en la plaza de Callao de 
Madrid. Se generó una gran expectativa y mucho contenido. Basándose en esta acción de marketing, el propio Fnac produjo esta película basada en la marca y donde, además del entorno en la que se desarrolla y que aparece constantemente, se muestran otra gran cantidad de marcas lo que produjo una influencia mayor que con un product placement.

- La Lego película. (2014). Es un claro ejemplo de branded content. Tras la película, Lego creó un videojuego basado en las aventuras narradas en la misma y ha dado continuidad a esta acción con productos, como juguetes inspirados en los personajes y merchandising.

FALSO TRAILER:

- Bad Night (2009). Dirigido por Alex de la Iglesia. A modo de cortometraje, el director realizó un falso tráiler sobre una supuesta película, "Bad Night" y que generó una enorme expectación. En realidad era un contenido original y llamativo que se creó para la Fundación de Ayuda contra la Drogadicción. Según el director, quisieron mantener la sensación de que se trataba de una película de terror real para conseguir la implicación de la juventud.

WEBSERIE:

- The Hire (2001-2002). Aunque considerado como estrategia advertainment, o como webserie, la producción de BMW es una estrategia que se basa en ocho cortometrajes y que contó con la dirección de directores como Tony Scott o Guy Ritchie. Entre ellos, destaca "Hostage" del director chino-estadunidense John Woo, por la que recibió el premio al mejor corto de acción en el Festival de cine de Los Ángeles. Los ocho cortometrajes (también entendidos como capítulos de webserie) son los siguientes:

- The Hire - Ambush: de John Frankenheimer.

- The Hire - Chosen: de Ang Lee.

- The Hire - Beat the devil: de Tony Scott.

- The Hire - Start: de Guy Ritchie.

- The Hire - Powder Keg: de Alejandro González Iñarritu.

- The Hire - Hostage: de John Woo.

- The Hire - Ticker: de Joe Carnahan.

- The Hire - The follow: de Wong Kar-Wai.

- La Huída (2012). Es una webserie que forma parte de una campaña branded content. Producida por la agencia de PR, Casanova se nutre de rostros muy conocidos del cine, la televisión y la cultura. Está rodada en Alicante para la marca Energy System que promociona. El 
cortometraje, de menos de media hora, está separado en tres webisodios.

- La otra película (2014): en una serie online (2014-actualidad), producida por "Sony Pictures España" y protagonizada por los YouTubers españoles El Rubius y Mangel, dónde parodian los últimos estrenos cinematográficos de la propia productora. (FILMAFFINITY).

- Entregados (2015). Desarrollada por UEstudio para Renault. La primera webserie producida por un grupo de prensa en España es una iniciativa basada en la colaboración del departamento de Branded Content de Unidad Editorial con la firma Renault. Unidad Editorial se suma al desarrollo de nuevos contenidos digitales con el lanzamiento de Entregados. Los capítulos de esta iniciativa podrán seguirse hasta diciembre de 2015 en la home y secciones de motor de MARCA.com y EXPANSION.com, además de los perfiles sociales de estas cabeceras y en la propia web del grupo Renault. Entregados narra, en clave de humor, la historia de Fermín, un joven repartidor que comienza a trabajar en la empresa de su futuro suegro Pascual, un excéntrico personaje con el que vivirá anécdotas de lo más peculiares. Sus historias, divididas en 24 capítulos, forman, a su vez, parte de un site en el que también se incluyen vídeos formativos enfocados a mejorar la motivación y la eficiencia en el día a día, tanto dentro como fuera del trabajo. Con el lanzamiento de esta webserie, refuerza su línea estratégica enfocada a la creación de contenidos novedosos, impactantes y orientados a su visualización en dispositivos móviles. http://www.unidadeditorial.es/noticia.aspx?id=3435

\section{FASHION FILM:}

- Lady Blue Shangay (2010). Dirigido por David Lynch para la Dior. La protagonista es Marión Cotillard y la música de Franz Ferdinaz.

- A Therapy (2012). Dirigido por Roman Polanski para la marca Prada. Está interpretado por Helena Bonham Carter y Ben Kingsley.

- Castello Cavalcanti (2013). Dirigido por Wes Anderson para la marca Prada.

\section{ESTRATEGIAS:}

- Intel y Toshiba: The beauty in side. Es una estrategia basada en una miniserie producida por Intel y Toshiba. Consta de seis cortometrajes y han creado una historia en la que se incluye a las personas que decidieron voluntariamente participar en el proyecto. Se aprovecha así la creación de contenido, la participación y la 
interactividad creando un universo nuevo. Es una estrategia valiente ya que conecta con el espectador en tres tiempos: la petición de participación, el visionado de los cortometrajes según se han ido exponiendo y en el producto final que se ha realizado uniendo todos los cortometrajes. Los seis capítulos pueden encontrarse en YouTube y la información técnica del proyecto en http://www.filmaffinity.com/es/film348546.html. En el año 2015 se realizó en Corea del Sur una película con el mismo argumento. http://www.filmaffinity.com/es/film336934.html.

- $\quad$ El Corte Inglés: La suerte de quererte. Es una estrategia dirigida por Roberto Pérez Toledo en la que se cuentan pequeñas historias de amor para el día de San Valentín. La campaña ofrece relatos interesantes, entretenidos y que pretenden que el espectador disfrute. Se accede a ellos a través del hashtag \#Lasuertedequererte, lo que pone de manifiesto que busca el apoyo en redes sociales. Los cortometrajes son los siguientes:

- Doce o trece tequieros al mes. https://www.youtube.com/watch?v=5Z3OLlC7VcM

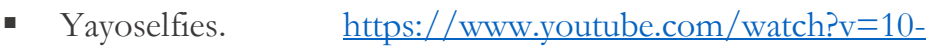
aEkmTHW4

- Equis

$\mathrm{O}$

corazón.

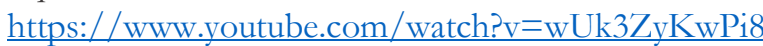

- Sí a todo. https://www.youtube.com/watch?v=T5fkH6JcH28

- Red Giant:

- Plot device (2012). Dirigido por Seth Worley, es un branded content para la marca Red Giant y para promocionar uno de sus productos: el Magic Bullet, que es un programa de etalonaje que hace que el protagonista cambie de una forma muy peculiar y original. Cada cambio de tonalidad implica un cambio de situación para el protagonista.

- Tempo (2012). Segundo cortometraje dirigido por Seth Worley para la marca Red Giant y para promocionar sus productos. En este caso, un científico debe evitar que una nueva y poderosa tecnología caiga en las manos equivocadas.

\section{- Liberty:}

- Incidencias navideñas (2014). Cortometraje producido por VCCP Spain para Liberty Seguros. Es una obra que obtuvo un gran éxito y fue reconocida con un bronce en los premios Inspirational, que organiza IAB Spain; y con plata y oro en FIAP (Festival Iberoamericano de la Publicidad). Fue creado 
para la Navidad de 2014 y ha sido muy reconocido en el sector publicitario

- Regalo (2016). Dirigido por Gracia Querejeta. Cortometraje que continúa con la campaña anterior y producido por VCCP Spain para Génesis. Es una acción de branded content que la compañía utiliza para felicitar a las madres en el "Día de la madre". Felicita a las madres en su día y homenajea a los asesores de su centro de servicios. Se distribuye a través de redes sociales y a través de su Oficina de Clientes. Esta fórmula potencia la marca a través de la cara visible de la aseguradora. Tiene una gran carga emocional y el mensaje se traduce en que una madre, nunca deja de ser madre. Se grabó en las oficinas del Grupo Líberty en Madrid.

- Gas Natural Fenosa: Cinergía es una estrategia de branded content gestionada por Gas Natural Fenosa http://cine.gasnaturalfenosa.es/cinergia/. Se han realizado tres ediciones y las dos primeras se han centrado en la fórmula del cortometraje. En la última se ha producido una película que se nutre de cuatro cortometrajes. Se promociona la búsqueda de la eficiencia energética y la importancia del ahorro.

- Primera edición 2014:

- Ultravioleta. Dirigido por Paco Plaza. Corto de Terror.

http://cine.gasnaturalfenosa.es/cinergia/primeraedicion/ultravioleta/

- $\quad$ 1:58. Dirigido por Rodrigo Cortés. Corto de terror y thriller.

http://cine.gasnaturalfenosa.es/cinergia/primeraedicion $/ 1-58 /$

- Inquilinos. Dirigido por Jaume Balagueró. Corto fantástico con un toque de humor. http://cine.gasnaturalfenosa.es/cinergia/primeraedicion/inquilinos/

- Domonic. Dirigido por Juan Cruz. Corto futurista. http://cine.gasnaturalfenosa.es/cinergia/primeraedicion/domonic/

- Segunda edición 2015:

- Vaca Paloma. Dirigido por Paco León. http://cine.gasnaturalfenosa.es/cinergia/segundaedicion/vaca-paloma/ 
- Consumo responsable (Nivel 7). Dirigido por Santiago Segura http://cine.gasnaturalfenosa.es/cinergia/segundaedicion/nivel-7/

- Beta. Dirigido por Josep Pujol. Para la creación de este cortometraje, Gas Natural Fenosa organizó un certamen entre estudiantes de cine y de comunicación audiovisual, Certamen Talento Cinergía.

http://cine.gasnaturalfenosa.es/cinergia/segundaedicion/beta/

- Un corazón roto no es como un jarrón roto o un florero. Dirigido por Isabel Coixet. http://cine.gasnaturalfenosa.es/cinergia/segundaedicion/un-corazon-roto/

- Tercera edición 2016: La iniciativa de Cinergía presenta su III edición con una novedad, la producción de un largometraje escrito por Daniel Sánchez Arévalo y realizado en forma de cuatro cortos entrelazados dirigidos por cuatro directores diferentes: Kike Maíllo, Borja Cobeaga, Alberto Ruiz Rojo (ganador del premio 'Talento Cinergía') y el propio Sánchez Arévalo. Este largometraje branded content ha sido presentado por todos los directores en compañía del director general de Comunicación y Gabinete de Presidencia, Jordi García Tabernero, el presidente de El Terrat Andreu Buenafuente, y los actores Michelle Jenner y Fernando Tejero. Un largometraje y cuatro cortos sobre eficiencia energética. El largometraje 'En tu cabeza' y los cuatro cortos a partir de los que se ha construido transmiten la importancia del ahorro y la eficiencia energética y están vinculados a los servicios de Gas Natural Fenosa. "Tú y dos veces yo", "Cabra y oveja", "Milagros y Remedios" y "Suegro y Suegra" se han estrenado en los principales festivales de cine del país.

\section{- Estrella Damm:}

- Vale (2015): dirigido por Alejandro Amenabar y protagonizado por Dakota Johnson y Quim Gutierrez. La estrategia de Estrella Damm consistió en realizar un spot para televisión que hacía referencia a poder visualizar el cortometraje de forma personal en la web de la marca. El corto, de más de doce minutos, cuenta una historia que se 
relaciona con la marca, más que con los emplazamientos de producto, que los hay, en el ambiente que se crea en la historia. El grupo que aparece puede relacionarse con el target específico de la marca de cerveza. Se realizan además múltiples referencias cinematográficas y musicales que buscan la identificación de un público determinado. En él se desarrolla la historia y, sobre todo al final, los protagonistas terminan bebiendo una Estrella Damm. Transmite los valores de la empresa y crea una atmósfera específica y busca la presencia sutil de la marca.

- Las pequeñas cosas (2016). Dirigido por Alberto Rodríguez y protagonizado por Jean Renó y Laia Costa. La marca ha creado una historia que atrapa al espectador desde el inicio y que consigue transportarlo a un film de verdad, de forma que no sienta que está viendo un anuncio de cerveza. El desarrollo de la historia es muy completo, tiene un buen guion y suscita interés. Utiliza la misma estrategia que en el cortometraje del año anterior y se realiza también un spot para televisión que deriva al espectador a un visionado posterior, individual y voluntario. Los enclaves son excelentes y contribuyen a arropar la historia y así facilitar el mensaje que la marca quiere transmitir.

\section{CORTOMETRAJES AUTÓNOMOS:}

- Carlota (2013). Dirigida por Nacho Vigalondo para la marca de cerveza Brabante. Pretende la campaña apoyar las distintas manifestaciones artísticas y que coincidan con los valores de la marca: orgullo, fuerza e independencia.

- La vuelta a la tortilla (2013): dirigida por Paco León para la marca de cerveza Buckler 0,0. Es un cortometraje solidario inspirado en ocho casos reales de mujeres que han sufrido un cáncer de mama. La recaudación de la obra va destinada a la Sociedad Española de Oncología Médica.

- El Xow (2014). Es un cortometraje producido por Attic Films sobre el restaurante DiverXO de Dabiz Muñoz. El objetivo es mostrar al cliente una experiencia única. Se trate de mostrar un universo gastronómico bajo el leit motiv de "vanguardia o morir". https://vimeo.com/107451752

- ¿Crees en el destino? (2016). Dirigido por Daniel Sánchez Arévalo para la marca Euromillones 2016. Cortometraje producido por 
Loterías y apuestas del estado que se incluye en la campaña "No hay nada más grande".

- 17 años juntos (2016). Dirigido por Javier Fesser para la marca ING Direct. ING DIRECT ha cumplido 17 años en España y para celebrarlo ha querido rendir un homenaje a sus clientes con el cortometraje 17 años juntos. El corto, ha sido dirigido por el director español Javier Fesser y está inspirado en la historia entre ING DIRECT y sus clientes. Una relación que empezó hace ya 17 años y que se fundamenta en el primer día gracias al compromiso y la pasión por las personas que tiene banco. Según María Alonso, Directora de Marketing de ING DIRECT: "el objetivo de esta acción es agradecer a todos y cada uno de nuestros clientes su confianza y fidelidad a lo largo de estos 17 años, en los que, sin duda, han sido parte fundamental del éxito del banco". https://www.ingdirect.es/sobreing/prensa/prensa280616.html\#

\section{Discusión y conclusiones}

El branded content surge para acercar la marca al consumidor de una manera sutil y casi oculta, pero la realidad es que, si la marca no aparece de ninguna forma, el recuerdo será menor. No se trata de agobiar con una aparición constante del producto o servicio que se ofrece, sino de que a través de una experiencia sensitiva, el usuario recuerde la marca. De hecho, si la presencia de la marca es excesiva e incluso forzada, puede impedir el desarrollo natural del discurso narrativo y reducir la eficacia de la estrategia.

En la mayoría de las ocasiones, en los escritos de referencia, se alude a la importancia de recibir una experiencia que se relaciona con una grata y agradable sensación, pero en el estudio que se ha realizado, se constata que el terror y el thriller son estrategias que sorprenden mucho al espectador y que tienen un alto poder de recuerdo.

Tal y como señala el estudio Conten Scope 2015, el 25\% de los anunciantes comenzará a realizar estrategias de branded content a largo plazo, es decir, que prevén desarrollar historias que enganchen al consumidor durante periodos largos de tiempo, ya que las experiencias autónomas tienen, en general, menor repercusión.

El formato más útil es el audiovisual. Ya sean productos más cortos, estilo spot, o más largos como cortometrajes e incluso películas, se posicionan para 
fomentar el recuerdo de la marca. Lo valioso es el storytelling, la historia que está detrás de la marca y a la que el usuario debe acceder de forma voluntaria.

Las redes sociales se posicionan como el mejor lugar donde se puede adquirir visibilidad para la marca. El fácil acceso y la posibilidad de compartir los recursos audiovisuales hacen de esta opción un canal perfecto para el desarrollo de las estrategias de branded content. Las marcas, y por ende las empresas y organizaciones, se vuelven más humanas y se socializan de cara al gran público.

En los primeros contactos del usuario con las fórmulas audiovisuales creadas bajo una marca determinada, podría parecer que los productos son de una calidad menor ya que a todos los usuarios se les puede considerar potenciales creadores de historias. La realidad es bien distinta y, en este estudio se ha detectado, que ante esta circunstancia, ocurre lo contrario y es que se produce una mayor profesionalización del sector audiovisual en beneficio del binomio contenido-marca. El sector, tanto de la realización como de la producción, se esfuerza para destacar a través de la calidad de sus productos.

El branded content ofrece un sinfín de posibilidades de comunicación entre la marca y la creatividad y, además, se ha observado que la presencia de la música es un referente común en las estrategias que utilizan la ficción. Este código sonoro sirve para potenciar la historia que se cuenta en los cortometrajes.

Las fórmulas diseñadas se realizan en formatos que puedan adaptarse a los diversos tipos de plataformas y pantallas desde los que se puede acceder al producto. Se detecta también que, en ocasiones, se realizan versiones adaptadas a la televisión, en formato spot, para poder llegar a dos tipos de públicos bien diferenciados. En el spot se hace alusión a la posibilidad de ver, de manera voluntaria, la historia completa.

La ficción en general y el cortometraje en particular, abren a la industria del cine una opción creativa que puede beneficiar de manera muy significativa a esta industria cultural. Desde el año 2010 en adelante, se observa que se ha producido un aumento en el número de cortometrajes utilizados para potenciar la imagen de marca.

Este incremento se acompaña y potencia además con la participación, cada vez más numerosa, de directores de reconocido prestigio así como actores y personajes famosos. Con su participación aumentan el prestigio, no solo del cortometraje sino también de la marca. 
El cortometraje destaca como una de las fórmulas más atractivas para desarrollar estrategias de branded content y, lo más importante, es que parece que lo seguirá siendo en el futuro.

\section{Referencias}

Antevenio.com (2016). Disponible en http://www.antevenio.com/blog/2016/03/6-ejemplos-de-campanas-debranded-content/

Caerols Mateo, R. y De la Horra Veldman, Y. (2015). Fórmulas creativas en la publicidad de moda. Un análisis de Madrid Fashion Film Festival y su impacto en redes sociales. Prisma Social no 14 (Jun-nov 2015), 336-378.

Castelló-Martínez, A.; Del Pino-Romero, C. y Tur-Viñes, V. (2016).

Estrategias de contenido con famosos en marcas dirigidas a un público adolescente. ICONO 14 (14), 123-154.

Catorce Producciones. Disponible en http://www.catorce.es/the-beautyinside-una-miniserie-de-toshiba-e-intel/ http://www.catorce.es/

Content Scope 2015 (2015). Disponible en http://prnoticias.com/podcast/ondacro/branded-content/20146444content-scope-2015-espana

Costa-Sánchez, C. (2014). Comunicación Corporativa Audiovisual. Nuevos formatos audiovisuales al servicio de la Comunicación Corporativa. XV Foro Universitario de Investigación en Comunicación. El nuevo diálogo social: organizaciones, públicos y ciudad. Foro.com 2014, Universidad de Vigo.

Costa-Sánchez, C. (2015). El cambio que viene, Audiovisual branded content. Telos (Cuadernos de Comunicación e Innovación), 1-9.

De Aguilera-Moyano, J.; Baños-González, M. y Ramírez-Perdiguero, J. (2015). Branded entertainment: los contenidos de entretenimiento como herramienta de comunicación de marketing. Un estudio de su situación actual en España. Revista Latina de comunicación Social, 70, 519-538.

Del Pino, C. y Olivares, F. (2007). Brand placemet y advertainment. integración y fusión entre la ficción audiovisual y las marcas. ZER, 22, 341-367. 
Del Pino-Romero, C. y Castelló-Martínez, A. (2015). La comunicación publicitaria se pone de moda: branded content y fashion films. Revista Mediterránea de Comunicación, 6 (1), 105-128.

Corazones de acero (FURY) (2014). Dirigida por David Ayer, USA, Sony Pictures Entertainment, Columbia Pictures, Qed International.

Galindo Rubio, F. (2004). Comunicación Audiovisual Corporativa. Cómo audiovisualizar la identidad de las organizaciones. Salamanca: Publicaciones Universidad de Salamanca.

Galindo Rubio, F. (2005). Comunicación Audiovisual Corporativa: un modelo de producción. Actas dos III SUPCOM, LUSOCOM e IBÉRICO, Universidade da Beira Interior, Covilha, 667-677.

Gambetti, RC. y Grafigna, G. (2010). The concept of engagement: a systematic analysis of the ongoing marketing debate. International Journal of Market Research, 52 (6), 801-826.

Grandío, M. (2015). Indicadores para la evaluación de la alfabetización transmedia en los estudios universitarios de Comunicación, en Peinado Miguel, F. (Coord.), Formación, perfil profesional y consume de medios de los alumnos en comunicación, Cuadernos Artesanos de Comunicación, 77, La Laguna (Tenerife). Latina

Jenkins, H. (2008). Convergence Culture. La cultura de la convergencia de los medios de comunicación. Barcelona, Ed Paidós.

La Huida (cortometraje) (2012), producido por Energy System Soyntec, S.A.

Lorán-Herrero, M.D. (2012). Análisis audiovisual de los spots de la campaña electoral autonómica 2011 en la Región de Murcia. En Rocío Zamora (Coord.) La imagen del candidato electoral en el contexto de la cibercampaña: las elecciones autonómicas de 2011 en la Región de Murcia. (pp. 87-122). Sevilla: Punto Rojo

Lorán-Herrero, M.D. (2016). La Comunicación Corporativa Audiovisual: propuesta metodológica de estudio. Tesis Doctoral. Universidad Católica de Murcia.

Matellanes Lazo, M. (2014). Nuevos formatos televisivos para no perder audiencias, Miguel Hernández Communication Journal, n5, 293-325. 
Reinares-Lara, E; Reinares-Lara, P. y Olarte-Pascual, C. (2016). Formatos de publicidad no convencional en televisión versus spots: un análisis basado en el recuerdo. Historia y Comunicación Social, Vol. 21, nº 1, 257-278.

Renedo, C. y Alonso del Barrio; E. (2014). Marketing de contenidos en los medios de comunicación: usos y aplicaciones. XV Foro Universitario de Investigación en Comunicación. El nuevo diálogo social: organizaciones, públicos y ciudad. Foro.com 2014, Universidad de Vigo, Pontevedra

Rodríguez, A.; Gil, S. y Marzal, J. (2015). La crisis del Habitar: Cine y publicidad en el Branded Content Cinergia-Gas Natural Fenosa. Questiones publicitarias, Vol. I, nº 20, 142-159.

Scolari, Carlos A. (2013). Narrativas Transmedia. Cuando todos los medios cuentan. Barcelona, Deusto.

Thesocialmediafamily.com (2016). Disponible en http://thesocialmediafamily.com/tendencias-branded-content/

Vivar Zurita, H. (2011). TIC, Internet y el sector de la comunicación. Nuevos perfiles profesionales para una comunicación digital. Telos, $\mathrm{n}^{\circ} 87$. Disponible en https://telos.fundaciontelefonica.com/url-direct/pdfgenerator?tipoContenido $=$ articuloTelos\&idContenido $=2011051109310001 \& \mathrm{i}$ $\underline{\text { dioma }=\mathrm{es}}$ 


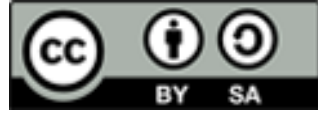

Licencia Creative Commons

Miguel Hernández Communication Journal

mhjournal.org

\section{Cómo citar este texto:}

María Dolores Lorán Herrero (2017): “The use of the short film as a branded content strategy", en Miguel Hernández Communication Journal, no8, pp. 153 a 177. Universidad Miguel Hernández, UMH (Elche-Alicante). Accessed mhjournal.org] 
